\title{
NÍVEIS DE ANTICORPOS ANTI-HBS EM ESTUDANTES E PROFISSIONAIS DE SAÚDE VACINADOS PARA HEPATITE B DE UMA UNIVERSIDADE DO INTERIOR PAULISTA
}

\author{
Ana Rita Paladino Tumitan, Ludmila Ichioka Silgueiro, Isabela Cassiano Gonçalves, Juliano de
} Sant'Ana Gasquez

Universidade do Oeste Paulista - UNOESTE, Curso de Farmácia, Presidente Prudente, SP. e-mail: anarita@unoeste.br

\begin{abstract}
RESUMO
O objetivo deste estudo foi avaliar os níveis de anticorpos anti-HBs entre estudantes e profissionais de saúde vacinados contra o vírus da hepatite $\mathrm{B}(\mathrm{VHB})$, bem como checar o nível de conhecimento sobre a necessidade de realizar o teste pós-vacinação. Noventa e dois participantes responderam o questionário sobre vacinação, forneceram dados de seus cartões vacinais e uma amostra de sangue. Os títulos de antiHBs foram determinados por quimioluminescência. As análises estatísticas foram realizadas através do teste de Qui-quadrado. Observou-se melhor resposta de anticorpo na faixa etária entre 20-29 anos e significativa queda dos níveis nos vacinados há mais de 15 anos. A maioria conhecia a necessidade de realização do teste pós-vacinação e $13 \%$ o fizeram. Os resultados corroboram com outros estudos e demonstram que os estudantes e profissionais de saúde precisam ser informados da importância de monitorar seus níveis de anticorpos, que deverá ser realizada antes do início de práticas que envolvam risco biológico.
\end{abstract}

Palavras-chave: hepatite B, vacinação, imunidade.

\section{ANTI-HBS LEVELS IN HBV-VACCINATED STUDENTS AND HEALTHCARE PROFESSIONALS FROM UNIVERSITY AT SÃO PAULO STATE}

\begin{abstract}
The aim of this study was to assess antibody against hepatitis B (anti-HBs) levels among students and healthcare professionals vaccinated for hepatitis B virus (HBV) and check their level of knowledge about the need to perform the test post-vaccination. Ninety-two participants answered a questionnaire about vaccination, provided data from their vaccination cards and blood sample. The anti-HBs level was determined by chemiluminescence. Statistical analyses performed by Chi-square test. We observe a better antibody response in an age group of 20-29 years and significant drop in levels in vaccinated more than 15 years. Most participants knew the necessity of post-vaccination test and $13 \%$ did so. These results corroborate with other studies, and demonstrate that students and healthcare professionals need information about the importance of monitoring their antibody levels, which accomplished before starting practices involving biological risk.
\end{abstract}

Keywords: hepatitis B, vaccination, immunity.

\section{INTRODUÇÃO}

A hepatite B é uma infecção viral, que pode causar doenças agudas e crônicas. Em todo o mundo estima-se que aproximadamente 780.000 pessoas morrem anualmente pela hepatite $B$, sendo 650.000 de cirrose e câncer de fígado devido a Hepatites crônicas e outros 130.000 por causa de hepatite aguda. O vírus da hepatite B (HBV) é transmitido através do contato com sangue e outros fluidos corporais de uma pessoa infectada ${ }^{1}$. Entre os grupos de risco, os profissionais de saúde são os mais suscetíveis de contrair hepatite B por sua vulnerabilidade a se acidentarem com materiais perfuro-cortantes ${ }^{2}$. O risco de contrair o VHB para profissionais de saúde é quatro vezes superior do que da 
população geral adulta ${ }^{3}$. A vacinação representa o principal instrumento para prevenir a infecção pelo $\mathrm{VHB}^{4,5}$, no entanto cerca de $10 \%$ das pessoas vacinadas não produzem anti-HBs suficiente após receber as três doses ${ }^{6,7}$. São considerados protegidos aqueles em que os níveis de anti-HBs forem $\geq 10 \mathrm{mUl} / \mathrm{mL}^{8}$.

Embora vários fatores do hospedeiro como tabagismo, obesidade, infecção pelo HIV e presença de doenças crônicas, possam contribuir para a diminuição da imunogenicidade na primeira série da vacina, a idade é o principal fator ${ }^{9}$. A série recomendada de três doses da vacina intramuscular para hepatite $B$ induz uma resposta protetora de anticorpos em mais de $90 \%$ dos adultos saudáveis com menos de 40 anos $^{10,11}$.

Em profissionais de saúde, quando a sorologia é negativa (anti-HBs $<10 \mathrm{mUl} / \mathrm{mL}$ ), passado muito tempo após a terceira dose, devese aplicar uma dose e repetir a sorologia um mês após. Caso positivo considerar imunizado, caso negativo completar o esquema com mais duas doses $^{12}$.

Existe, portanto, a necessidade de sensibilizar os profissionais e estudantes da área da saúde sobre a importância da vacinação contra a hepatite $B$ e do monitoramento de seus níveis de anti-HBs.

O objetivo deste estudo foi avaliar os níveis de anticorpos anti-HBs em estudantes e profissionais de saúde vacinados para hepatite $B$ de uma universidade do interior paulista, bem como verificar a relação dos títulos de anticorpos com sexo, idade, esquema de vacinação, tempo decorrido após a última dose da vacina e checar o nível de conhecimento dos participantes sobre a necessidade da realização do teste após a vacinação.

\section{METODOLOGIA}

Foi realizado um estudo transversal, evolvendo estudantes e profissionais de saúde de uma universidade do interior paulista, com idade de 18 anos ou mais, que tinham recebido as três doses ou mais da vacina para hepatite $B$ e que concordaram em participar da pesquisa, por meio de um termo de consentimento livre e esclarecido. $O$ estudo foi aprovado pelo Comitê de Ética em Pesquisa (CAAE n. 50662915.7.0000.5515).

Os 92 participantes, incluíram 79 estudantes dos cursos de Farmácia e Biomedicina, 7 professores, 4 funcionários do
Laboratório de Análises Clínicas e 2 funcionários de outros setores da universidade. Entre eles, 17 $(18,5 \%)$ pertenciam ao sexo masculino e 75 $(81,5 \%)$ pertenciam ao sexo feminino. A idade dos sujeitos da pesquisa variou de 18 a 59 anos, com média de 22,9 anos.

Os participantes responderam um questionário sobre vacinação e forneceram dados de sua carteira vacinal. Foi colhida uma amostra de cinco mililitros de sangue de cada participante, obtida por punção venosa. As amostras de sangue foram centrifugadas a 2000 rpm, por dez minutos. Após centrifugação, os soros foram separados, devidamente identificados e mantidos a $-20^{\circ} \mathrm{C}$, até o momento da realização do teste. Os soros foram testados para titulação de anticorpos anti-HBs por quimioluminescência, utilizando o sistema de imunoensaio ADVIA Centaur ${ }^{\circledR}$ CP (Siemens Heathcare GmbH Henkestr. 127, 91052 Erlangen Geermany), de acordo com as instruções do fabricante.

Os participantes foram agrupados segundo a faixa etária em 5 grupos: < 20 anos, 20-29 anos, 30-39 anos, 40-49 anos e $\geq 50$ anos. Quanto a titulação de anti-HBs, os participantes foram classificados em 3 grupos: 0-9 $\mathrm{mUl} / \mathrm{mL}, 10$ $99 \mathrm{mUl} / \mathrm{mL}$, e $\geq 100 \mathrm{mUl} / \mathrm{mL}$. Outra classificação com base nos título de anti-HBs $<10 \mathrm{mUl} / \mathrm{mL}$ (sem soroproteção) e $\geq 10 \mathrm{mUl} / \mathrm{mL}$ (soroproteção), foi feita para as análises.

Foram determinadas a média dos títulos de anti-HBs; a taxa de soroprotegidos e não soroprotegidos; a taxa de respondedores, hiporespondedores e não respondedores. A análise estatística para verificar a relação dos títulos de anti-HBs com gênero, esquema de vacinação, faixa etária e tempo decorrido após a última dose da vacina, foi realizada utilizando o teste de Qui-quadrado e o valor de $p<0,05$ considerado significativo.

Os dados obtidos no questionário foram analisados para verificar o nível de conhecimento sobre a necessidade da realização do teste após vacinação.

\section{RESULTADOS}

A média dos títulos de anti-HB foi 225,43 $\mathrm{mUl} / \mathrm{mL}$ e taxa de soroproteção foi de $75 \%$. Observamos uma boa resposta de anti-HBs ( $\geq 100$ $\mathrm{mUl} / \mathrm{mL}$ ) - respondedores, em $43,5 \%$, uma baixa resposta (10-99 $\mathrm{mUI} / \mathrm{mL}$ ) - hiporespondedores, em $31,5 \%$ e ausência de resposta $(<10 \mathrm{mUl} / \mathrm{mL})$ 
- não respondedores em $25 \%$ dos participantes. A relação dos títulos com gênero, calendário de vacinação, faixa etária e tempo decorrido após a última dose da vacina estão representadas nas Tabelas 1, 2, 3 e 4, respectivamente.

Tabela 1. Níveis de anti-HBs de acordo com o gênero.

$p=0,9367$

$$
\text { Anti-HBs }
$$

\begin{tabular}{llll} 
Gênero & $<10 \mathrm{mlU} / \mathrm{mL}$ & $\geq 10 \mathrm{mlU} / \mathrm{mL}$ & Total \\
\hline Masculino & $4(23,5 \%)$ & $13(76,5 \%)$ & $17(100 \%)$ \\
Feminino & $19(25,3 \%)$ & $56(74,7 \%)$ & $75(100 \%)$ \\
Total & $23(25 \%)$ & $69(75 \%)$ & $92(100 \%)$ \\
\hline
\end{tabular}

Tabela 2. Níveis de anti-HBs de acordo com o esquema de vacinação.

$p=0,7498$

\begin{tabular}{llll}
\hline $\begin{array}{l}\text { Esquema de } \\
\text { vacinação }\end{array}$ & \multicolumn{4}{c}{ Anti-HBs } \\
\hline Regular & $11(29,7 \%)$ & $26(70,3 \%)$ & $37(40,2 \%)$ \\
Irregular & $12(23,1 \%)$ & $40(76,9 \%)$ & $52(56,5 \%)$ \\
llegível & $0(0 \%)$ & $03(100 \%)$ & $03(3,3 \%)$ \\
Total & $23(25 \%)$ & $69(75 \%)$ & $92(100 \%)$ \\
\hline
\end{tabular}

Tabela 3. Níveis de anti-HBs de acordo com a faixa etária.

\begin{tabular}{|c|c|c|c|c|}
\hline \multirow{2}{*}{$\begin{array}{l}\text { Faixa etária } \\
\text { (anos) }\end{array}$} & \multicolumn{2}{|c|}{ Anti-HBs } & \multirow{2}{*}{ Total } & \multirow{2}{*}{$p$} \\
\hline & $<10 \mathrm{mIU} / \mathrm{mL}$ & $\geq 10 \mathrm{mIU} / \mathrm{mL}$ & & \\
\hline$<20$ & $19(37,3 \%)$ & $32(62,7 \%)$ & $51(55,4 \%)$ & 0,8793 \\
\hline $20-29$ & $4(13 \%)$ & 27 (87\%) & $31(33,7 \%)$ & $0,0137^{*}$ \\
\hline $30-39$ & $0(0 \%)$ & $1(100 \%)$ & $1(1,1 \%)$ & 0,5183 \\
\hline $40-49$ & $0(0 \%)$ & $6(100 \%)$ & $6(6,5 \%)$ & 0,1283 \\
\hline$\geq 50$ & $0(0 \%)$ & $3(100 \%)$ & $3(3,3 \%)$ & 0,3062 \\
\hline Total & $23(25 \%)$ & $69(75 \%)$ & 92 (100\%) & 0,0788 \\
\hline
\end{tabular}

* Diferença significativa.

Tabela 4. Níveis de anti-HBs de acordo com o tempo decorrido após vacinação.

\begin{tabular}{lcccc}
\hline \multirow{2}{*}{$\begin{array}{l}\text { Tempo após } \\
\text { vacinação (anos) }\end{array}$} & \multicolumn{2}{c}{ Anti-HBs } & Total & p \\
\cline { 2 - 3 }$<5$ & $010 \mathrm{mIU} / \mathrm{ml}$ & $\geq 10 \mathrm{mlU} / \mathrm{ml}$ & & $4(4,4 \%)$ \\
$5-10$ & $0(0 \%)$ & $4(100 \%)$ & 0,03203 \\
$11-15$ & $7(15,2 \%)$ & $39(84,8 \%)$ & $46(50 \%)$ & 0,6776 \\
$>15$ & $16(45,7 \%)$ & $19(54,3 \%)$ & $35(38 \%)$ & $0,0008^{*}$ \\
llegível & $0(0 \%)$ & $3(100 \%)$ & $3(3,2 \%)$ & - \\
Total & $23(25 \%)$ & $69(75 \%)$ & $92(100 \%)$ & $0,0127^{*}$ \\
\hline
\end{tabular}

* Diferença significativa.

Os dados a respeito do conhecimento dos participantes sobre a necessidade de realizar o teste anti-HBs após vacinação estão demonstrados na Tabela 5. 
Tabela 5. Conhecimento sobre a necessidade de realizar o teste anti-HBs pós-vacinação e sua realização segundo a categoria profissional.

\begin{tabular}{lcc}
\hline \multicolumn{3}{c}{ Conhecimento sobre a necessidade de realização do teste anti-HBs } \\
\hline Categoria profissional & Sim & Não \\
\hline Estudante & $45(57 \%)$ & $34(43 \%)$ \\
Professor & $7(100 \%)$ & $0(0 \%)$ \\
Funcionário do laboratório & $4(100 \%)$ & $0(0 \%)$ \\
Funcionário de outros setores & $2(100 \%)$ & $0(0 \%)$ \\
Total & $58(63 \%)$ & $34(37 \%)$ \\
\hline \multicolumn{3}{c}{ Realização do teste anti-HBs } \\
\hline Categoria profissional & $\mathrm{Sim}$ & $\mathrm{Não}$ \\
\hline Estudante & $5(6,3 \%)$ & $74(93,7 \%)$ \\
Professor & $6(85,7 \%)$ & $1(14,3 \%)$ \\
Funcionário do laboratório & $1(25 \%)$ & $3(75 \%)$ \\
Funcionário de outros setores & $0(0 \%)$ & $2(100 \%)$ \\
Total & $12(13 \%)$ & $80(87 \%)$ \\
\hline
\end{tabular}

\section{DISCUSSÃO}

No presente estudo, foi verificada taxa de soroproteção de $75 \%$, semelhante ao valor relatado por Jha et al. ${ }^{13}$ e por Sacchetto et al. ${ }^{14}$ que encontraram $73,5 \%$ e $79,2 \%$ respectivamente, mas menor do que as taxas demonstradas por outros autores, ${ }^{2,15-17}$. Essas diferenças podem ser devido ao tempo decorrido após a vacinação. Em nosso estudo, as dosagens de anti-HBs foram realizadas entre 1 a 19 anos após a última dose da vacina, enquanto no estudo de Nashibi et al. ${ }^{17}$ relatando $94,1 \%$ de soroproteção foram realizadas entre 1 a 6 meses após a última dose. Neste estudo, em participantes que receberam três doses da vacina, observamos $43,5 \%$ de respondedores (anti-HBs $\geq 100 \quad \mathrm{mUl} / \mathrm{mL}$ ), $31,5 \%$ dos hiporespondedores (anti-HBs-99 $10 \mathrm{mUl} / \mathrm{mL}$ ) e ausência de resposta (anti-HBs $<10 \mathrm{mUl} / \mathrm{mL}$ ) em $25 \%$ dos participantes. As taxas de respondedores e hiporespondedores foram similares as encontradas por Shooshtari et al. ${ }^{16}$ de $56,4 \%$ e $30,8 \%$, respectivamente. De acordo com a Vigilância Epidemiológica do Estado de São Paulo ${ }^{8}$, profissionais de saúde com sorologia negativa (anti-HBs $<10 \mathrm{mUl} / \mathrm{mL}$ ), passado muito tempo após a terceira dose, devem receber uma dose extra e repetir a sorologia após um mês. Os participantes não soroprotegidos (25\%) identificados em nosso estudo foram orientados para procurar uma Unidade de Saúde com seus resultados para receber o reforço necessário. Não houve diferenças significativas nos níveis de anti-HBs em relação ao gênero $(p=0,9367)$ e ao esquema de vacinação $(p=0,7498)$. Quanto ao gênero, nossos resultados corroboram com os estudos de Fagundes \& Tabalipa $^{18}$, Rezaei et al. ${ }^{19}$ e Shooshtari et al. ${ }^{16}$. As irregularidades na administração das doses parece não interferir na resposta de anticorpos, se a série de vacinação é interrompida após a primeira dose, a segunda deve ser administrada logo que possível. A segunda e a terceira dose devem ser separadas por um intervalo de 2 meses, se apenas a terceira dose está atrasada, deve ser administrada a qualquer momento ${ }^{20}$.

Encontramos uma melhor resposta de anti-HBs em indivíduos na faixa etária de 20-29 anos $(p=0,0137)$, concordante com o resultado de Shooshtari et al. ${ }^{16}$. Acima de 40 anos, há um declínio na imunogenicidade e com 60 anos, apenas $75 \%$ das pessoas vacinadas desenvolvem níveis protetores de anti-HBs ${ }^{10,18,21}$. Houve diminuição de anti-HBs com tempo após a vacinação, sendo significativa a queda nos títulos de anticorpos no grupo dos vacinados há mais de 15 anos $(p=0,0008)$. Estes resultados são semelhantes aos relatados em outros estudos, embora tenham sido realizados com populações diferentes ${ }^{19,22,23}$.

Quanto ao conhecimento dos participantes sobre a necessidade da realização do teste anti-HBs teste após a vacinação, notamos que, embora $63 \%$ dos participantes afirmassem ter conhecimento da necessidade de sua realização, apenas $13 \%$ fizeram. Entre os alunos, $57 \%$ tinham conhecimento, mas apenas 6,3\% fizeram (Tabela 5). Estes últimos valores são semelhantes aos relatados por Sacchetto et al. ${ }^{14}$ 
em um estudo realizado com estudantes de odontologia.

Os resultados desse estudo mostraram que, embora a maioria dos estudantes de farmácia e biomedicina esteja ciente da necessidade de monitoramento de seus títulos de anti-HBs pós-vacinação, uma pequena porcentagem o realizou. As análises sorológicas mostraram alunos não soroprotegidos, após a conclusão da vacinação.

Esses achados implicam na necessidade de realização de campanhas de educação sobre a importância do monitoramento dos níveis de anticorpos anti-HBs, que deve ser feito antes do início de práticas que envolvam riscos biológicos. As limitações desse estudo são o número de participantes da pesquisa e o envolvimento de discentes de apenas dois cursos da área da saúde. Novos estudos devem ser realizados com maior número de voluntários envolvendo estudantes dos cursos como medicina, enfermagem, fisioterapia, odontologia, além dos estudantes de farmácia e biomedicina.

\section{AGRADECIMENTOS}

Aos participantes da pesquisa e à responsável técnica do laboratório de Análises Clínicas da Universidade do Oeste Paulista, Lígia Maria Delfino Caldeira.

\section{CONFLITO DE INTERESSES}

Os autores declaram não haver qualquer potencial conflito de interesse que possa interferir na imparcialidade deste trabalho científico.

\section{REFERÊNCIAS}

1. World Health Organization. Hepatitis B. Fact sheet N²04 Updated March 2015. Accesso em: 11 Abr 2017. Disponível em: http://www.who.int/mediacentre/factsheets/fs204/e $\mathrm{n} /$

2. Ciorlia LAS, Zanetta DMT. Hepatitis B in healthcare workers: prevalence, vaccination and relation to occupational factors. Braz J Infect Dis. 2005;9(5):3849. DOI: http://dx.doi.org/S1413-86702005000500005

3. Byrne EB. Viral hepatitis: on occupational hazard of medical personnel. Experience of the Yale-New Haven Hospital, 1952-65. JAMA. 1966;195:362-4. DOI: http://dx.doi.org/10.1001/jama.1966.0310005007002 $\underline{1}$
4. Ferreira CT, Silveira TR. Hepatites virais: aspectos da epidemiologia e da prevenção. Rev Bras Epidemiol. 2004;7:473-87. DOI: http://dx.doi.org/10.1590/S1415790X2004000400010

5. Lewis JD, Enfield KB, Sifri CD. Hepatitis B in healthcare workers: Transmission events and guidance for management. World J Hepatol. 2015;7(3):488-97. DOI: https://doi.org/10.4254/wjh.v7.i3.488

6. Leuridan E, Van Damme P. Hepatitis B and the need for a booster dose. Clin Infect Dis. 2011; 53:68-75. DOI: https://doi.org/10.1093/cid/cir270

7. Damme PV, Herck, KV. A review of the long-term protection after hepatitis $A$ and $B$ vaccination. Travel Med Infect Dis. $\quad 2007 ; 5(2): 79-84 . \quad$ DOI: https://doi.org/10.1016/j.tmaid.2006.04.004

8. Brasil. Ministério da Saúde. Manual dos Centros de Referência para Imunobiológicos Especiais. 4.ed. Brasília: Ministério da Saúde; 2014. 160p.

9. Averhoff F, Mahoney FJ, Coleman PJ, Schatz G, Hurwitz E, Margolis, HS. Risk factors for lack of response to hepatitis $B$ vaccine: a randomized trial comparing the immunogenicity of recombinant hepatitis B vaccine in adult population. Am J Prev Med. 1998;1:73-7.

10. Roome AJ, Walsh SJ, Cartter ML, Hadler JL. Hepatitis B vaccine responsiveness in Connecticut public safety personnel. JAMA. 1993;270:2931-4. DOI: https://doi.org/10.1001/jama.1993.03510240043029

11. Wood RC, MacDonald KE, White KE, Hedberg CH, Hanson M, Osterholm MT. Risk factors for lack of detectable antibody following hepatitis $B$ vaccination of Minnesota health care workers. JAMA. 1993;270:2935-72.

DOI: https://doi.org/10.1001/jama.1993.03510240047030

12. Centro de Vigilância Epidemiológica "Prof. Alexandre Vranjac" Divisão de Imunização. Divisão de Hepatites. Coordenadoria de Controle de Doenças. Secretaria de Estado da Saúde de São Paulo $\mathrm{DI} / \mathrm{DH} / \mathrm{CVE} / \mathrm{CCD} / \mathrm{SES}-\mathrm{SP}$, Vacina contra hepatite B. Rev Saude Publica. 2006; 40(6):1137-40. DOI: http://dx.doi.org/10.1590/S0034$\underline{89102006000700026}$

13. Jha AK, Chadha S, Bhalla P, Saini S. Clinical Study Hepatitis B Infection in Microbiology Laboratory Workers: Prevalence, Vaccination, and Immunity Status. Hepat Res Treat. 2012; 2012:1-5. DOI: http://dx.doi.org/10.1155/2012/520362 
14. Sacchetto MS LS, Barros SSLB, Araripe TA, Silva AM, Faustino SKM, Silva JMN. Hepatitis B: knowledge, vaccine situation and seroconversion of dentistry students of a public university. Hepat Mon. 2013;13(10):1-7.

DOI:

http://dx.doi.org/10.5812/hepatmon.13670

15. Gankzak MA. Cross-Sectional Study on Anti Hepatitis B Immune Status in Vaccinated Healthcare Workers in the West Pomeranian Region of Poland. Hepat Mon. 2012;12(3):185-9. DOI: http://dx.doi.org/10.5812/hepatmon.850

16. Shooshtari MHS, Makvandi M, Rasti M, Neisi N, Rastegarvand N, Pouremamali A, Haj MS, Ghaedi F. Evaluation of hepatitis $B$ surface antibody and specific gamma interferon response in health care workers after vaccination. Jundishapur J Microbiol. 2015;8(1):1-5.

DOI:

http://dx.doi.org/10.5812/ijm.13801

17. Nashibi R, Alavi SM, Yousefi F, Salmanzadeh S, Moogahi S, Ahmadi F, Farashahinejad M. Postvaccination Immunity Against Hepatitis B Virus and Predictors for Non-responders Among Medical Staff. Jundishapur J Microbiol. 2015;8(3):1-5. DOI: https://doi.org/10.5812/jjm.19579

18. Fagundes GD, Tabalipa FO, Silva J. Antibody levels in children after 10 years of vaccination against hepatitis B: a Brazilian community-based study. Rev Soc Bras Med Trop. 2012;45(2):260-2. DOI: http://dx.doi.org/10.1590/S0037-

\section{4}

19. Rezaei $M$, Nooripoor S, Ghorbani R, Ramezanshams F, Mamishi S, Mahmoudi S. Seroprotection after hepatitis $B$ vaccination in children aged 1 to 15 years in central province of Iran. J Prev Med Hyg. 2014;55(55):1-3.

20. Atkinson W, Humiston S, Wolfe C, Nelson R. Centers for Disease Control and Prevention Epidemiology and Prevention of Vaccine-Preventable Diseases. $5^{\text {th }}$ ed. Washington D.C: Public Health Foundation; 1999.

21. Wood RC, Macdonald KE, White KE, Hedberg CH, Hanson M, Osterholm M T. Risk factors for lack of detectable antibody following hepatitis $B$ vaccination of Minnesota health care workers. JAMA. 1993;270(24):2935-22. DOI: http://dx.doi.org/10.1001/jama.1993.0351024004703 $\underline{0}$
22. Van Der Sande M A B, Waight PA, Mendy M, Zaman S, Kaye S. Long-term protection against HBV chronic carriage of gambian adolescents vaccinated in infancy and immune response in HBV booster trial in Adolescence. PloS One. 2007;2(8):e753. DOI: https://doi.org/10.1371/journal.pone.0000753

23. Gilca V, De Serres G, Boulianne N, Murphy D, Ouakki M, De Wals $P$, Trudeau G, Massé R, Dionne $M$. Long-term persistence of immunity after vaccination of pre-adolescents with low doses of a recombinant hepatitis B vaccine. Hum Vaccin Immunother. 2013;9(8):1685-90. DOI:

http://dx.doi.org/10.4161/hv.25015

Recebido para publicação em 11/07/2017

Revisado em 20/04/2018

Aceito em 18/06/2018 